\title{
Generalized Predictive Control of Nonlinear Systems Based on Genetic Algorithm
}

\author{
Zhong-liang Shao \\ Guangdong Technical College of Water Resources and Electric Engineering \\ Guangzhou,China,510635 \\ veday@163.com
}

\begin{abstract}
Aiming the traditional predictive function control can't control the nonlinear and changeful object effectively, the disadvantages of obtaining control in approximate ways are analyzed, and the optimality criterion based on genetic algorithm is proposed in this paper. During the simulation of a heat exchanger, genetic algorithm and the two approximate ways are used to get control respectively, and then the control effects of the three methods are compared. Simulation results show that predictive controller solving based on the genetic algorithm can enable the system output to better track the reference input and the change of controlled quantity to be more stable.
\end{abstract}

Keywords-nonlinear system; generalized predictive control; genetic algorithm

\section{INTRODUCTION}

A number of practical industrial processes, due to its complexity, are difficulty for us to achieve the mechanism model through theoretical derivation. In this case, it is of great important to identify models through experimental data. Among lots of non-linear experimental models, Hammerstein model and Wiener model are quite widely used.

Aiming at this kind of models, the paper studies a kind of non-linear predictive control method on the basis of genetic algorithm optimization strategy, fit for non-linear systems with special structure such as Hammerstein model, Wiener model and Hammerstein-Wiener model. Such kind of model is commonly used in the process industries. Thus, it has a practical value. This greatest advantage of this method is simple in computation. Compared to GPC algorithm, it just adds a process of solution of non-linear equation and its realtime of control can be guaranteed. For some which are difficult to extract a root by using analytic method, this essay also provides two approximate ways. In this article, the simulation results of several cases indicate that generally the receding-horizon algorithm based on genetic algorithm could achieve quite satisfying control effects; while for a few objects with complicate non-linear relationship, the approximate way cannot guarantee to control their stability.

\section{HAMMERSTEIN MODEL PREDICTIVE CONTROL BASED ON TWO-STEP METHOD}

Most of industrial controls are equipped with non-linear characteristic. However,as for such kind of system predictive control,due to lack of effective mathematic tools and the difficulty of establishing accurate predictive models, there is no research method which is commonly used and effective.

Hammerstein model can be used to describe a non-linear system that is connected in series by static non-linear subsystem and dynamic non-linear subsystem, which is a kind of quite common experimental model of the non-linear system. The document [3] adopts the relevant conclusions of the wide predictive control (GPC) based on Hammerstein model, offers the design approach of the controller and conducts the analysis. The document [1] and document [2] is aiming at the root of non-linear equation, discussing whether the controller could come true and providing two approximate ways.

However,the above ways all rely on the detailed form of non-linear function. Neither could it guarantee its good control accuracy nor could it guarantee its realization of control quantity. This essay analyzes the disadvantages of these approximate ways and introduces a kind of recedinghorizon algorithm based on two-step method. Via stimulation, it proves the validity and feasibility of this strategy.

The generalized predictive model of linear subsystem of Hammerstein model is:

$$
Y_{p}(k+1)=G \Delta X(k)+H \Delta X(k-1)+F Y(k)
$$

The key point of predictive control is the rolling optimization. And the optimization result should be the optimal control quantity which is able to put into effect. It is noted that formula (1) only shows the linear part of the whole model. Despite of easy to solve, it cannot directly get the control quantity. For Hammerstein model, there is a natural idea that showing $\Delta \mathrm{X}$ with $\Delta \mathrm{U}$ expression to acquire a completed predictive model and further get a performance index about $\Delta \mathrm{U}$ function. Finally, we take the way of vector derivation to get the optimal control law.

\section{TWO-STEP METHOD RECEDING-HORIZON ALGORITHM}

In order to make full use of existing conclusions, it can adopt the receding-horizon algorithm based on two-step method, separating the linear part and non-linear part. The linear part takes GPC. Taking the performance index of the iterative schemes: 


$$
J=\left\|Y_{p}(k+1)-Y_{r}(k+1)\right\|_{Q}^{2}+\|\Delta X(k)\|_{R}^{2}
$$

Put formula (1) into formula (2), the result is

$$
\begin{aligned}
& J=\left[G \Delta X(k)+H \Delta X(k-1)+F Y(k)-Y_{r}(k+1)\right]^{T} Q[G \Delta X(k) \\
& \left.+H \Delta X(k-1)+F Y(k)-Y_{r}(k+1)\right]+\Delta X^{T} R \Delta X
\end{aligned}
$$

Formula (3) takes the derivative of unknown control quantity $\Delta X(k)$, if $\partial J / \partial \Delta X=0$, the optimal control law gotten is:

$$
\begin{gathered}
\Delta X_{0}(k)=\left[G^{T} Q G+R\right)^{-1} G^{T} Q\left[Y_{r}(k+1)-H \Delta X(k-1)-F Y(k)\right] \\
x(k)=f(u(k))=\sum_{i=0}^{L} r_{i} u^{i}(k)
\end{gathered}
$$

The next step is getting the optimal control quantity $U(k)$ through the optimization result of the intermediate variable $\Delta X_{0} \quad(k)$. According to the information known $X_{0}(k)=X(k-1)+\Delta X_{0}(k)$ and the corresponding component between $U(k)$ and $X_{0}(k)$ satisfying function relation(5), the optimal control quantity $U(k)$ could be achieved through formula (5). If the control horizon $M>1$, it totally needs to solve equation (5) M times to get $U(k)$. Under this condition, the structure of the whole control system is displayed like Figure 1 as follows:

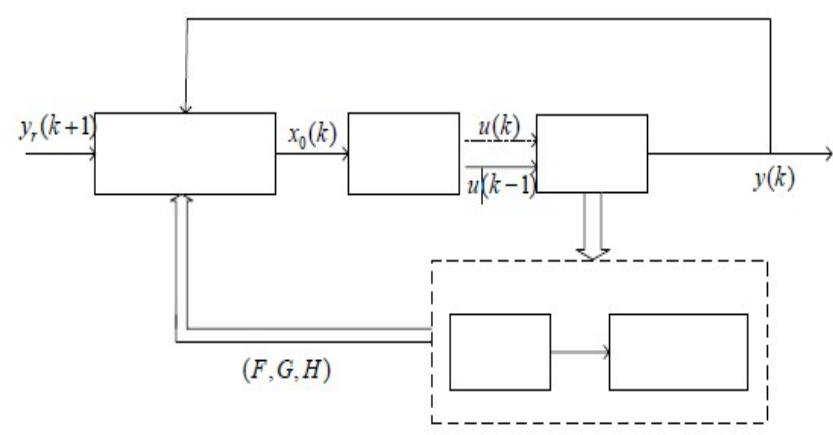

Figure 1 The principle figure of two-step method GPC of Hammerstein model

\section{THE APROXIMATION WAY OF SOLVING THE CONTROL QUANTITY}

Selecting the quadratic performance index used in document [2] and [4], shown by the weighted norm:

$$
J=\left\|Y_{p}(k+1)-Y_{r}(k+1)\right\|_{Q}^{2}+\|\Delta X(k)\|_{R}^{2}
$$

The optimal control quantity is achieved with formula (6) put and taking a derivative of $\mathrm{J}$ on the vector $\Delta X(k)$ :

$$
\begin{aligned}
& \Delta X_{0}(k)=\left(G^{T} Q G+R\right)^{-1} G^{T} Q\left[Y_{r}(k+1)-H \Delta X(k-1)-F Y(k)\right] \\
& x(k)=f(u(k))=r_{0}+r_{1} u(k)+\Lambda+r_{L-1} u^{L-1}(k)+r_{L} u^{L}(k)
\end{aligned}
$$

After getting intermediate variable $\Delta x_{0}(k)$, it must search for the corresponding control quantity $u_{0}(k)$. According to formula (8), it is an $\mathrm{L}$ order algebraic equation. It has certain difficulty to directly solve equation (8), may bringing out some new problems, such as the even-order without real root and control quantity not satisfying the physics restriction. Because of this, documents [1],[2] and[3] take two approximation solving ways.

Approximation 1: If $\mathrm{L}$, the highest order of equation (2), is a even number, when it removes the term including $L$ powers, the equation (8) becomes the odd-order equation with the highest order as L-1.

$$
x_{0}(k)=f_{1}\left(u_{1}(k)\right)=r_{0}+r_{1} u_{1}(k)+\Lambda+r_{L-1} u_{1}^{L-1}(k)
$$

In such way, at least, one real root $u_{1}$ could be acquired.

Approximation 2: replacing $u(k)$ in the higher-order term by $u(k-1)$ known, the solving equation is

$x_{0}(k)=f_{2}\left(u_{2}(k)\right)=r_{0}+r_{1} u_{2}(k)+r_{2} u^{2}(k-1)+\Lambda+r_{L} u^{L}(k-1)$

Thus, it could take advantage of the information ahead of time to calculate $u_{2}$.

Because of the mismatch among $f_{1}, f_{2}$ and $f$, it will have errors among the control quantities $u_{1}, u_{2}$ and $u_{0}$ achieved by adopting approximation ways. After such $u_{1}$ and $u_{2}$ are put into the actual objects, the $X$ will not meet the optimal control quantity (7). The essence of causing errors is the problem of mismatch between the model and object. The degree of mismatch is related to the approximation way and correlation parameter. The literature [1] makes a analysis on the errors from the perspective of internal mode control. The literature [2] adds the restriction of the intermediate variable into the control.

When we utilize the approximations above, the solving of $x_{0}(k)$ and $u_{0}(k)$ are relatively independent. Therefore, it is a kind of typical two-step strategy. It is noticed that although equation (9) and equation (10) are able to achieve a control effect, they cannot solve the following two difficult points. First, the control quantity gotten via the approximation ways might not satisfy the physics restriction, that is, the control quantity couldn't be carried out. Secondly, the optimization process doesn't include the restriction to the control quantity $u(k)$. Provided the practical problem needs to guarantee the stable change of control quantity, the optimal control rule (7) cannot meet the requirement ${ }^{\mathbf{I} \mathbf{1}}$. To solve two contradictions above, the paper proposes the optimization strategy based on the genetic algorithm. 


\section{THE OPTIMIZATION STRATEGY BASED ON GENETIC ALGORITHM}

The genetic algorithm is the optimal technology on basis of the evolution theory. It completely runs in accordance with the natural selection mechanism, with little dependence on the detailed form of solving problems.

We select the control quantity $U(k)$ as the decision variable. According to the demand of control horizon length, it totally chooses $\mathrm{M}$ control quantities to make up the unit. Because there are lots of variable numbers and the accuracy required is quite higher, it is suggested that we use floatencoding. The advantages of direct control quantity $U(k)$ encoding lie on two points. Firstly, the coded value is the actual value of the decision variable. The physics restriction condition of the control quantity that system requires can be limited via encoding, without attaching other measures such as penalization function. Secondly, after getting $U(k)$, it is easy to count $X(k)$. In this way, the root computation of equation (8) could be avoided.

Selecting the objective function as

$$
J=\left\|Y_{p}(k+1)-Y_{r}(k+1)\right\|_{Q}^{2}+\|\Delta U(k)\|_{R}^{2}
$$

Adding the restriction of $\Delta U(k)$ into the objective function can be effective to guarantee the stable change of the control quantity.

After adopting the genetic algorithm, the structure of the whole predictive control system is shown like figure 2. Due to directly encoding the control quantity and taking the performance index as the optimal object, the control quantity solving based on genetic algorithm actually could be regarded as a kind of completed solving strategy.

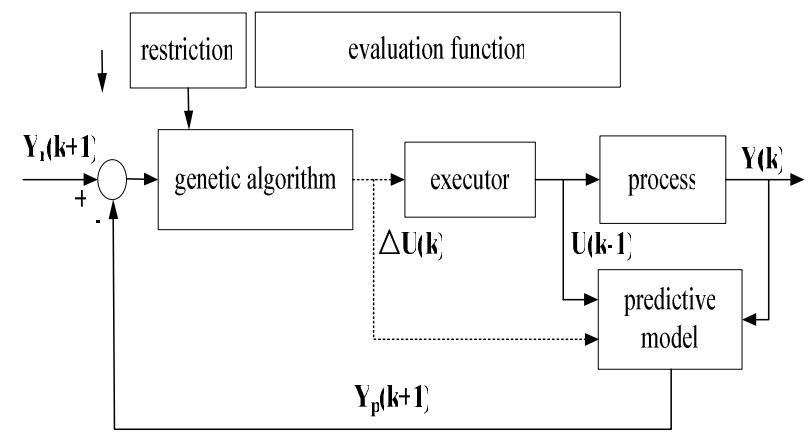

Figure 2: The predictive control process figure based on genetic algorithm

The strong point of adopting genetic algorithm to get control quantity is that it never has the problem of model mismatch, which could achieve the optimal root in theory. However, in fact, due to considering the demand of real-time, it cannot take quite huge population size and long evolutionary time. Hence, it could not ensure that the genetic algorithm could be converged to the optimal point. It might appear the prematurity phenomenon, which will cause certain errors. In order to try to reduce these errors, when we use genetic algorithm to get the control quantity, all initial population each moment is not generated with the random method. Part of individual could be produced by adopting the mutation test based on the answer achieved a moment before. What's more, the group must consist of the optimal control sequence gotten in a previous moment. And the rest take the random generating method for complement. In this way, at the same time when we make sure the stability of algorithm, it can also improve the efficiency of algorithm ${ }^{[5]}$.

\section{STIMULATION ANALYSIS}

It studies the heat exchanger problem always encountered in the industrial applications. Through the parameter reorganization, the heat exchanger could be introduced by using the following Hammerstein model ${ }^{[6]}$ :

$$
y(k)=\frac{0.312 z^{-1}-0.1587 z^{-2}}{1-1.732 z^{-1}+0.7143 z^{-2}} x(k)
$$

$$
x(k)=-30.352 u(k)+39.648 u^{2}(k)-30.17 u^{3}(k)+70.454 u^{4}(k)
$$

The parameter of the predictive control model is as follows: the optimal horizon length is 5; the control horizon length is 4 and the error weight coefficient is 1 . The control weight coefficient is 450 (The gain is quite big from $u$ to $X$ ). The flexibility coefficient of the reference trajectory is 0.4 ; the range of control quantity value is $[0,1]$. Choosing the whole stimulation horizon length as 80 sampling instants, three different output values are given in succession to fully check the algorithm performance.

First of all, we don't take into consideration the physics restriction of the control quantity, regarding the initial horizon value as the zero and adopting approximation 1 and 2 to conduct simulation counting. The output curve we get is what the figure 3 and 4 show.

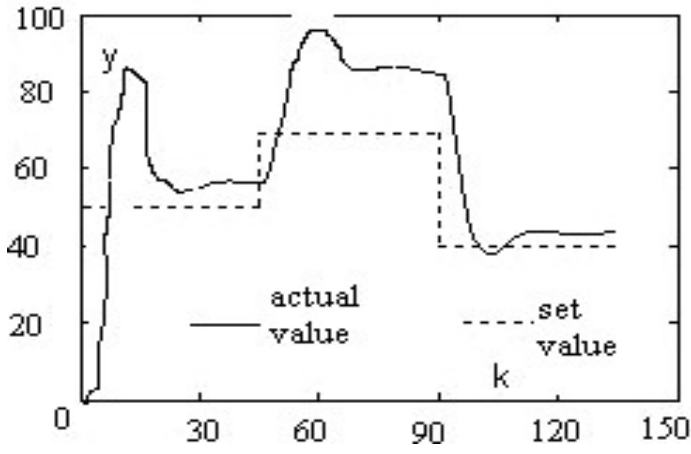

Figure 3: Output quantity curve (approximation 1) 


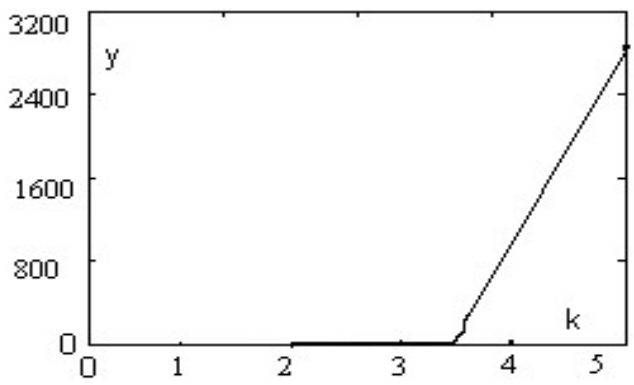

Figure 4: output quantity curve (approximation 2)

It is seen that approximation 2 makes the control fail and the approximation 1 manages to get the control with an effort but has great errors. Its effect is not ideal. Once adding the physics restriction of the control quantity, both approximation ways completely cannot control. The basic reason is that equation (11) has the highest reps. The coefficient of the highest-order power is high, which leads the approximate nonlinear relationship of equation (8) and (9) to have big differences from the actual situation, like figure 5:

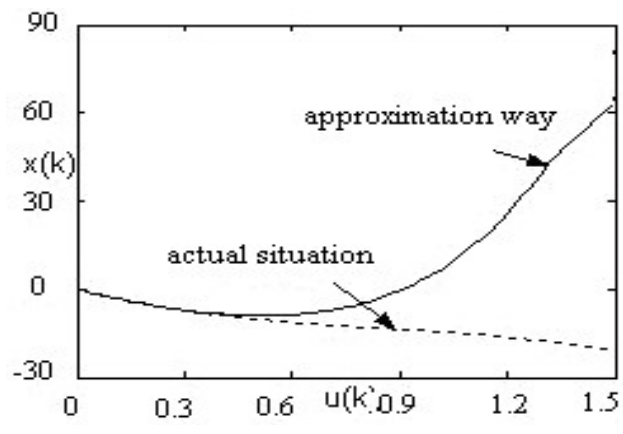

Figure 5: non-linear function comparison

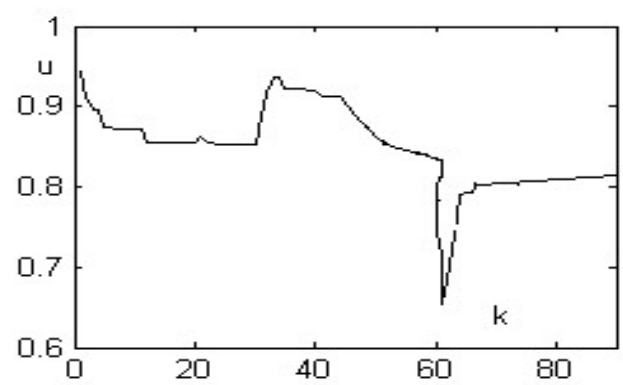

Figure 6: control quantity curve (genetic algorithm)

Now,we take the standard genetic algorithm to count. When we take detailed implementation, we adopt the optimal selection strategy, uniform arithmetic crossover and uniform mutation. The population size is 50 ; the crossover probability is 0.7 ; the mutation probability is 0.1 and the largest evolution is 50 generation. Owing to the minimum value problem of the root solving, the fitness function is Fitness $=C-J$. C is a constant. It needs to comprehensively think about the $\mathrm{Q}$ and $\mathrm{R}$ values which are achieved through attempts for many times. The control quantity curve and output quantity curve gotten by means of genetic algorithm are seen in figure 6 and 7 .

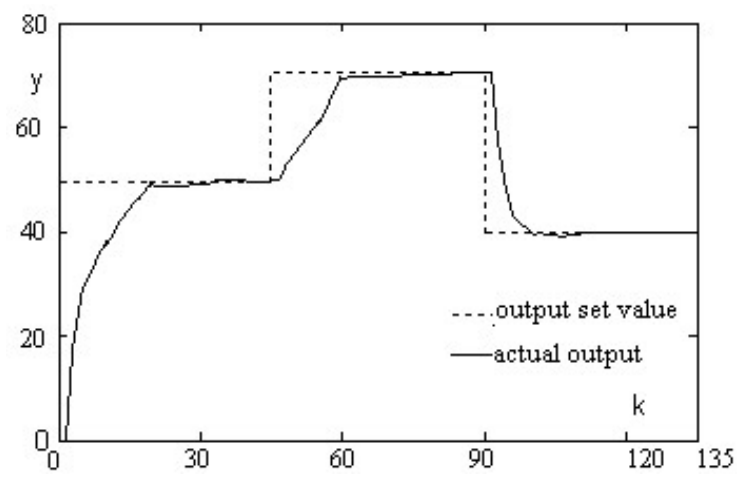

Figure 7: output quantity curve (genetic algorithm)

\section{CONCLUSION}

Seen from figure 7,compared with two approximation ways, the predictive controller solving based on the genetic algorithm can enable the system output to better track the reference input and the change of controlled quantity to be more stable. Meanwhile, as a kind of whole solving strategy, genetic algorithm has little dependence on the detailed form of non-linear relationship, without limitation to the low-order polynomial function. Therefore, its fitness range is wider.

It cannot be denied that the rise on this performance is at the cost of counting with more costs. Yet, PH neutralization process and heat exchanger process described with the Hammerstein model usually have quite large time constant. And, the demand on the rapidity is not high. Therefore, the receding-horizon algorithm based on the genetic algorithm is suitable for the online solving control quantity.

\section{REFERENCES}

[1] Xu Xiangyuan \& Mao Zongyuan. Analysis and study of predictive control based on Hammerstein model [J]. Control theory and applications, 2000, 17(4), 529-532.

[2] Ding Bao-cang, Li Shao-yuan. Design and analysis of Hammerstein nonlinear control systems with constraints[J].Control and Decision, 2003,18(1):24-28.

[3] Wang W. Generalized Predictive Control of Nonlinear Systems of the Hammerstein Form [J]. Control Theory and Applications (in Chinese), 1994, 11(6): 674-680.

[4] $\mathrm{Xu}$ Lei. Nonlinear predictive control and its simulation study based on Hammerstein/Wiener models [ D ]. Changsha: Hunan University, 2004.

[5] Zhang F. The Schur complement and its applications[M].New York:Springer, 2005

[6] Al-Duwaish H and Naeem, Wasif. Nonlinear Model Predictive Control of Hammerstein and Wiener Models Using Genetic Algorithm [A]. Control Applications, 2001. (CCA '01). Proceedings of the 2001 IEEE International Conference, on 5-7 Sept, 2001, 465-469. 